\title{
EDUCATION
}

\section{Defining the educational needs of recent dental graduates preparing for the Membership of the Faculty of Dental Surgery examination}

\author{
J. H. Bennett, ${ }^{1}$ E. R. Bennett, ${ }^{2}$ J. Lowry ${ }^{3}$ and J. Derry ${ }^{4}$
}

"An
individual's
approach to
learning will
evolve from a
dependence
on the
institution,
the teachers
and the course
structure
towards
greater
independence
and
autonomy"

\footnotetext{
$1^{*}$ Unit of Oral and Maxillofacial Pathology, Eastman Dental Institute, University College London. ${ }^{2}$ Department of History, University College London. ${ }^{3}$ Faculty of Dental Surgery, Royal College of Surgeons of England. ${ }^{4}$ The London Knowledge Lab, Institute of Education, London.

*Correspondence to: Dr Jon Bennett, Unit of Oral and Maxillofacial Pathology, Eastman Dental Institute, University College London, 256 Grays Inn Road, WC1X 8LD. Email: j.bennett@eastman.ucl.ac.uk
}

Objectives: On graduation, UK dentists wishing to advance their career enter two years of general professional training aimed at consolidating their undergraduate experience. The Membership of the Faculty of Dental Surgery examination (MFDS) attests to its successful completion and is a pre-requisite for entry into training programmes which lead to specialist status. Most MFDS candidates prepare for the examination on their own while in full-time employment and many reinforce this self-directed learning with participation in short revision courses or through distance learning. Here we seek to obtain data on the specific educational needs of these individuals.

Methods: Questionnaires were used to interrogate 92 UK graduates attending short MFDS revision courses of up to 1 week's duration to identify which topic areas were perceived as particular areas of weakness. To gain greater insight into the responses obtained, 18/92 respondents were selected at random and followed up with semi-structured interviews informed by the questionnaires.

Results: Basic medical science, human diseases, law and ethics and health and safety regulations were the areas of weakness most frequently highlighted by the respondents. Most had undergone comprehensive courses in the first two topics; however, the interviews suggested that this was generally in the early stages of undergraduate training when they had difficulty in contextualising large quantities of new information. In the case of the latter two, teaching had been very varied and several interviewees felt that it had been inadequate.

Conclusion: Recent graduates preparing for MFDS have clear educational needs. These data have begun to characterise the requirements of this group and may inform the planning of short revision courses designed to assist them.

\section{INTRODUCTION}

In common with medicine, dentistry has had to evolve to meet the challenges of technical advance. This has led, over a period of years to a shift from a situation in which a new dental graduate was considered to be qualified for a lifetime of practice to that today, in which the undergraduate years are regarded as the start of a continuum, leading into a period of vocational or general professional training, preparation for practice as a specialist or in primary care and participation in ongoing continuing professional development (CPD). The latter has now been formalised by the General Dental Council in its 'Lifelong Learning' scheme which is now mandatory for every dentist. Progression from one stage to the next depends on achieving defined training objectives and generally involves formalised examination and assessment.

Since its inception in 1998 the MFDS examination has become widely accepted as a benchmarking process, attesting to the satisfactory completion of a period of postgraduate general professional experience prior to entry into higher specialist or other areas of training. It is designed to be completed two years after graduation and to mark the completion of a period of structured application and consolidation of material learned as an undergraduate. The examination is currently set in three parts, Parts A, B and $\mathrm{C}$, is general in content and covers a wide range of topics drawn from the clinical dental disciplines, applied basic science, human disease, law, ethics, clinical governance and management-related issues applicable to the recent dental graduate.

Candidates usually prepare for the examinations on their own or seek the assistance of one of an increasing number of short courses or distance learning opportunities which may be part specific, or provide an integrated programme equally applicable to all parts of the examination. Despite their growing number there is a surprising lack of literature relating to the MFDS examination, and little information is available to inform course providers about the educational needs and concerns of revision course participants.

In this study we address this by seeking information about specific topic areas that prospective MFDS candidates perceive as giving most cause for concern, and to gain insight into what they would 


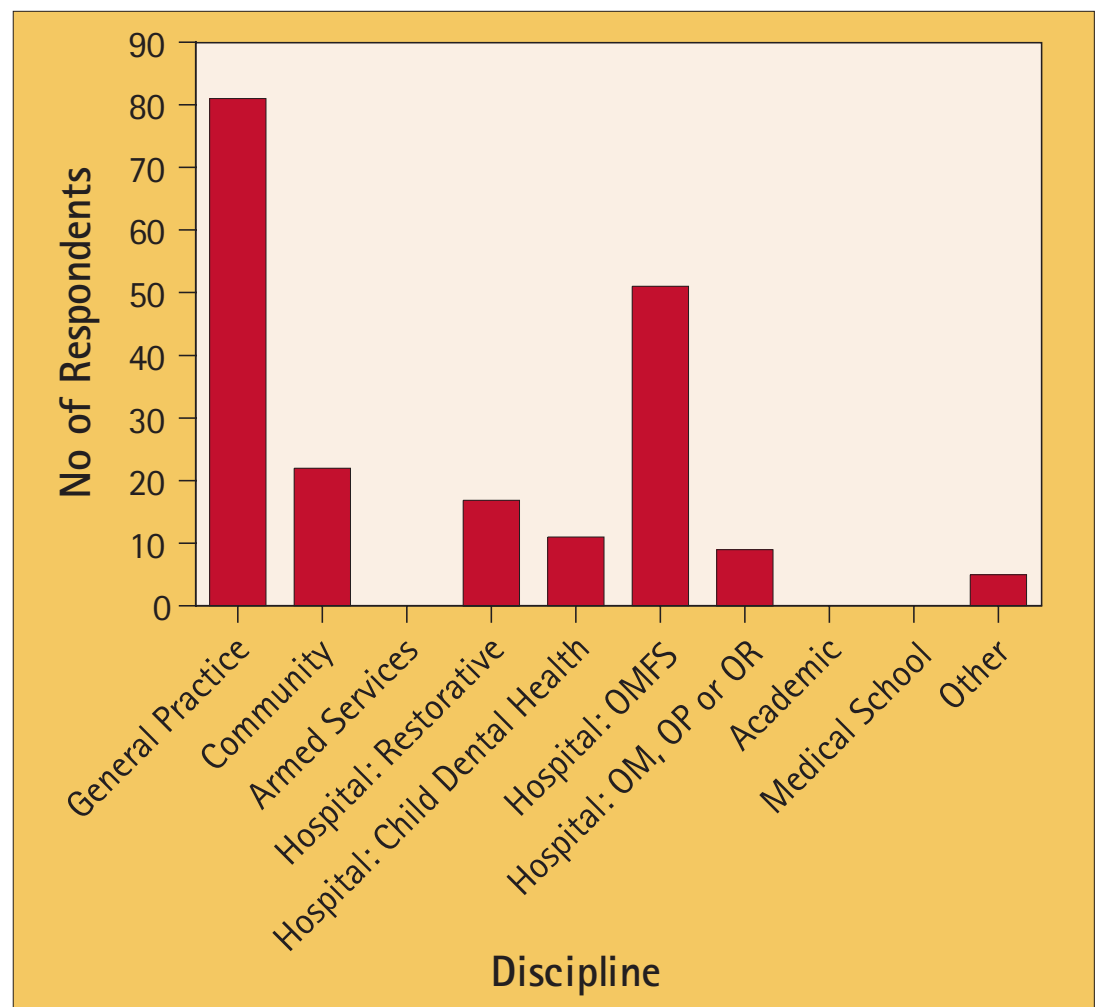

Fig. 1 Clinical Experience of Respondents

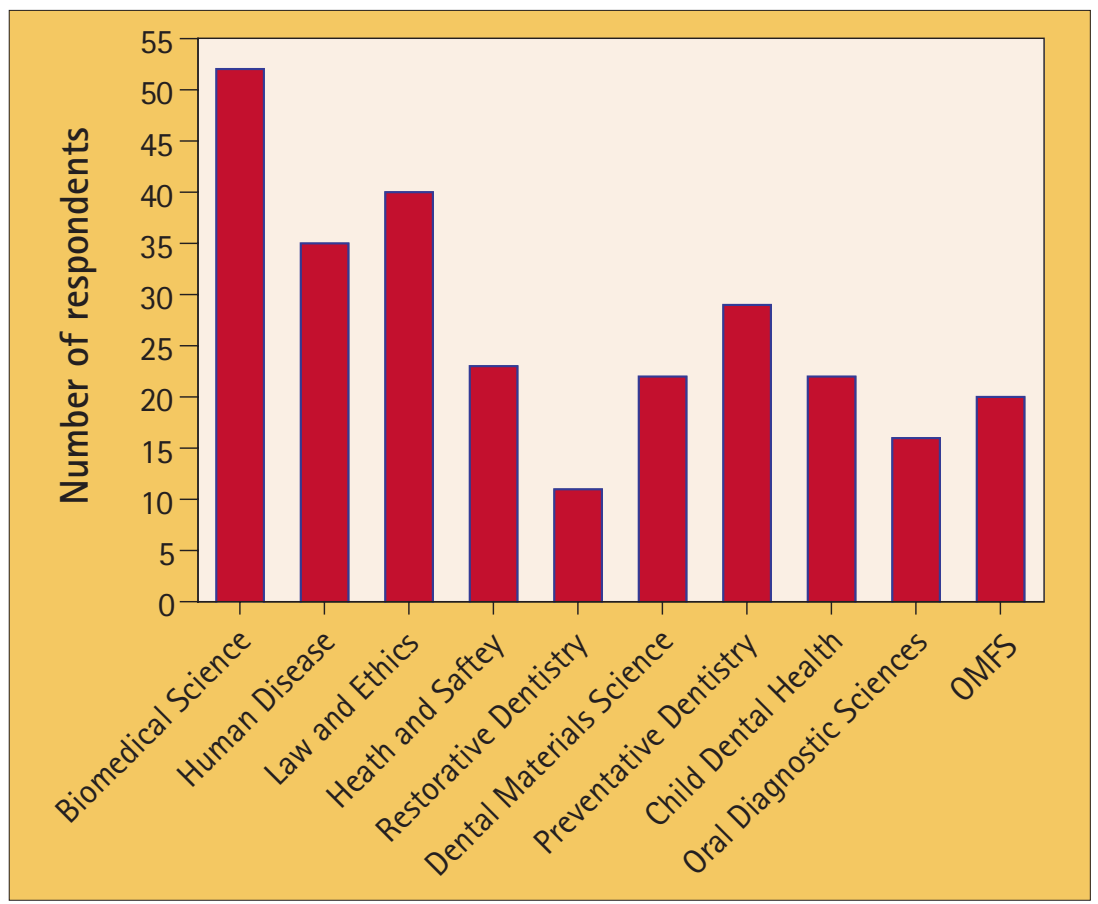

Fig. 2 Areas of weakness identified by individual participants

most like to see covered in short preparatory revision courses.

\section{MATERIALS AND METHODS \\ Inclusion criteria}

This study targeted dentists attending short dedicated revision courses in preparation for the MFDS examination at five centres between November 2003 and April $2004(n=92)$. Inclusion was restricted to dentists graduating from UK or Irish dental schools between 1998 and 2003 preparing for any part of the examination.

\section{Questionnaire}

The study was divided into two parts. For the first, a questionnaire was developed. This was divided into two sections, A and B. Section A collected basic biographic and demographic information with details of individual work experience since graduation. Section B was also divided into two parts. The first comprised a list of ten core subjects included in the dental curriculum (Table 2-Column $\mathrm{A}$ ) with its design informed by the GDC's education framework report: The first five years. ${ }^{1}$ Respondents were asked to prioritise the three topic areas from those listed which they felt caused them most concern (Table 2-Column B). In the second part they were asked to consider these three topic areas to indicate which of the component subjects making up each were considered to be problematic. Collection of these data took place under strictly controlled conditions just before the start of each course and was aimed at reducing the possibility of any unintentional bias arising out of the subject matter to be discussed.

\section{Semi-structured interview}

Eighteen respondents, selected at random from those who had agreed to provide contact details, were followed up with a semi-structured telephone interview. These were guided by the semi-structured questions outlined in Table 1, which were informed by the responses to the questionnaire, and were designed to explore in greater detail factors which influenced their choice of responses. A single interviewer did all the interviews. In most cases these were recorded and a full and accurate transcription of the text prepared. This was a time consuming, painstaking but essential process which allowed detailed review of the whole interview by others in an attempt to minimise interviewer bias. Although, because of the ease of obtaining high quality recordings for subsequent transcription, the telephone interview was a particularly effective tool, there were occasions when it was impossible to obtain those of an adequate quality. In these circumstances details were recorded as handwritten notes.

\section{Statistical analysis}

Professional statistical advice was obtained, the outcome of which was that as the data were essentially descriptive detailed statistical analysis was not considered appropriate.

\section{RESULTS}

\section{Baseline data}

All 92 individuals who fulfilled the criteria for inclusion in the study completed the questionnaire and had a mean age of 25.9 (range 22-43) years and a female: male ratio of 2.2:1. Every UK and Irish dental school was represented albeit with a bias to those in the southern part of the UK. Information relating to the undergraduate dental school entry qualifications of 75 participants was obtained. Seventy two, including the mature graduates, reported having traditional entry qualifications including GCE 'A' levels, Scottish Higher's or the Irish Matriculation. The remaining three were graduates from overseas who had come to 
the UK or Eire to obtain their primary dental qualification. No participant reported gaining admission as a graduate entrant.

\section{Postgraduate experience}

Most respondents $(n=60)$ were in their second year after graduation. Most, 88\% ( $\mathrm{n}=81)$ had experience of general practice as vocational trainees with 17 reporting sequential experience in both general practice and the community service, 18 had only worked in general practice and of these, 14 were in their first post-graduation year. The remainder had completed house officer, senior house officer or equivalent recognised posts for periods of three months or longer in $1.2 \pm 0.8$ (range 1-3) dental specialties (Fig. 1). These were dominated by oral and maxillofacial surgery (OMFS) appointments.

\section{Perceived areas of weakness}

Three perceived areas of weakness were identified by each participant, collated and summarised in Figure 2. Basic medical science was of concern to the greatest number of individuals (52/92) followed by law and ethics and human disease which concerned 40/92 and 35/92 of respondents respectively. Other topic areas, including preventative dentistry, health and safety issues and dental materials science were perceived as areas of weakness by 28, 23, 22 participants respectively. The more clinically orientated disciplines of oral surgery, the oral diagnostic sciences and restorative dentistry were of least concern overall and worrying, in turn, to 20,16 and 11 respondents.

Table 2 details which components of each subject area were causing concern. In this regard it is important to note that respondents were able to identify multiple component subjects where appropriate within each of their three chosen topic areas. Thus, the number of responses indicated in the Table, in many cases exceeded those given in Figure 2.

Considering an example, 52 participants expressed concern about biomedical sciences. Of these, 18 highlighted oral biology, 28 physiology, 26 biochemistry and 31 anatomy, indicating that some respondents were concerned about multiple areas of basic science with anatomy, physiology and biochemistry featuring more prominently than oral biology. Within human diseases, pharmacology and therapeutics emerged as a cause for particular concern. Those expressing under confidence in child dental health were more concerned about mastering an understanding of facial growth in contrast to the more applied areas of paediatric dentistry (Table 2). In the oral diagnostic sciences there was suggestion of a bias towards oral pathology as opposed to the more clinically orientated areas of oral medicine and radiology but the small numbers made this difficult to interpret. Despite its position as a core dental discipline, a relatively small number of course participants were worried by the restorative disciplines. While health and safety regulations, law and ethics and preventive dentistry caused several respondents some concern it was not possible to discern any obvious trends towards anxiety about one or other subdivisions of these areas.

\section{Table 1 Summary of questions used to guide semi-structured interviews}

- Do you think that teaching in this area received as an undergraduate met your subsequent needs?

- Did it stimulate further reading and 'self directed' learning?

- Did it give you a breadth and depth of understanding outside that required to pass assessments?

- Did it have relevance and/or immediacy to problems you encountered on graduation?

- Do you feel that, at an early stage of your undergraduate course when you were introduced to many new and important topic areas, you were simply not aware of what was relevant and what was not?

- Following on from this:

Would you have preferred more practical teaching?

Would you have preferred more teaching of a more formal lecture-based nature?

Would a 'problem based' approach have been helpful?

- Could this topic have been timetabled for a more appropriate part of the undergraduate course-if so, which?

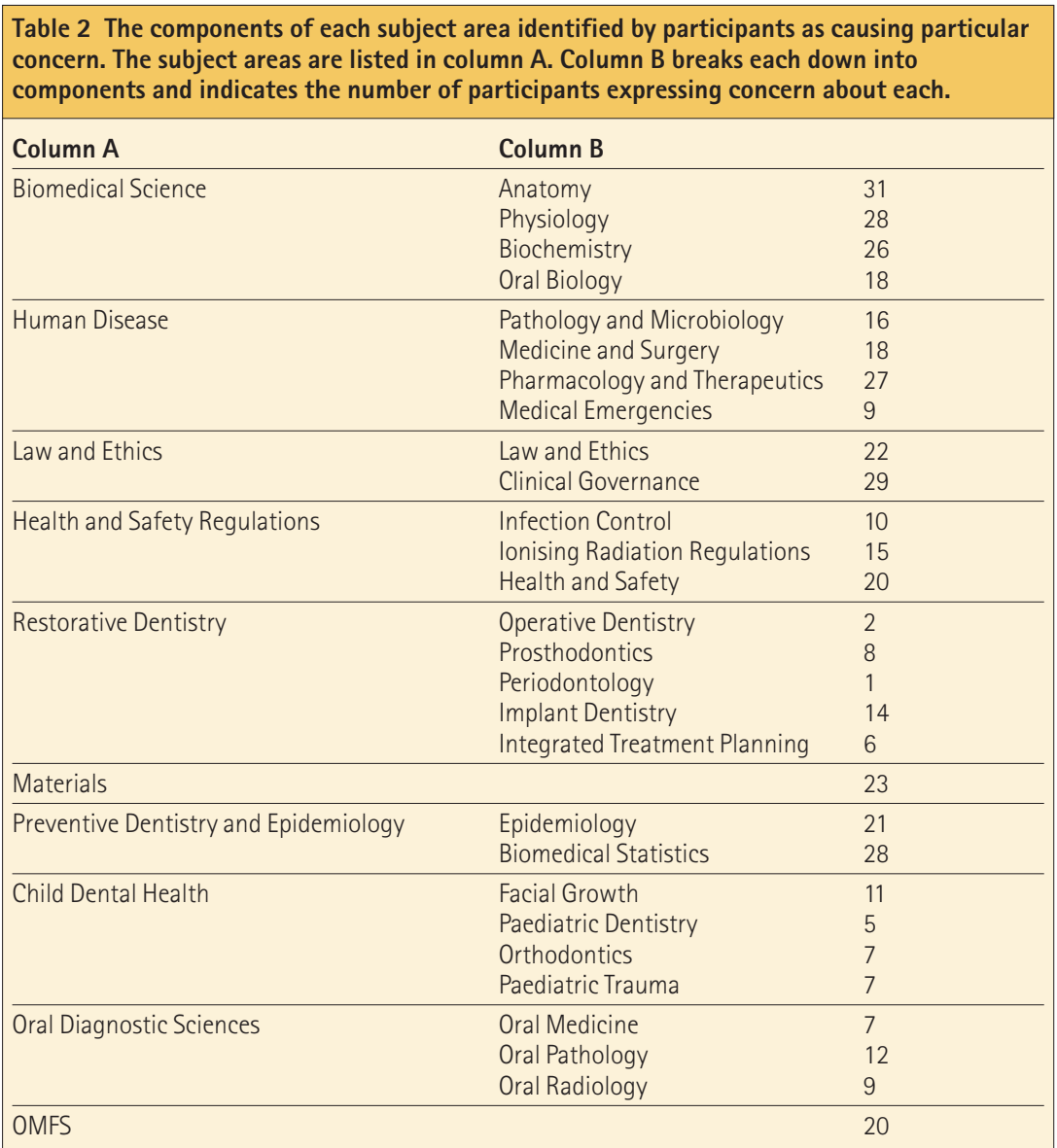

\section{Semi-structured interviews}

Every respondent asked agreed to give an interview. Each meeting lasted up to half an hour. Because of their qualitative nature and the scope of responses obtained they were considered as part of the discussion.

\section{DISCUSSION}

This study has helped to define the educational needs of dental graduates from UK dental schools preparing for the MFDS examination. Although partially based on quantitative data, education takes place in 'complex and ever changing networks of social interaction', ${ }^{2}$ and approaches based on the rigorous analysis of quantitative data may lead to key issues being overlooked. ${ }^{3}$ For example, in a systematic review comparing problem-based learning (PBL) with more traditional methods of course delivery, 
"On beginning independent practice, subjects which appeared 'dry' at dental school suddenly become interesting and relevant" examination performance, used as an index of outcome, could not demonstrate a difference between the two approaches. ${ }^{4}$ However, this fails to take into account a key objective of PBL, the development of self directed learning skills in preparation for ongoing participation in the learning process-a desirable outcome which is difficult to quantify and only becomes apparent some years later. To allow for this, a hybrid approach was adopted in which baseline quantitative data was collected and used to inform semi-structured interviews, a technique favoured in social science research. ${ }^{5}$

Any work of this sort is open to the challenge that it may not be representative. The study enjoyed a compliance rate of $100 \%$ and no obvious bias was detected. Nevertheless, some groups that have traditionally regularly generated a small number of MFDS candidates were not represented (eg the armed forces), so the possibility that a particular perspective may have been overlooked remains. In spite of this, we consider that this group represented a reasonable spectrum of opinion of UK graduates entering for the examination and that the results constitute a valid commentary on their particular needs.

Whilst all the topics listed on the questionnaire were perceived as areas of weakness by some respondents, the basic medical sciences (BMS), law and ethics and to a lesser extent human diseases (HD) stood out. This concern extended to all areas of BMS. In human disease, they were slanted towards pharmacology and therapeutics, pathology and microbiology as distinct from the more clinically applied areas of medicine, surgery or the management of medical emergencies.

It is not clear why this should be so. Educational theory might provide an answer. Applied biomedical science, a term used here to embrace both BMS and aspects of $\mathrm{HD}$, is a dominant feature of the early years of every UK and Irish dental curriculum. During the course, an individual's approach to learning will evolve from a dependence on the institution, the teachers and the course structure towards greater independence and autonomy, where individuals assume much more responsibility for their own learning. ${ }^{6}$ To put it simply, in the early stages of their course undergraduate students are not as skilled 'learners' as they become towards its conclusion. Consequently, material learned in the early stages may be less well retained and be harder for the student to apply.

A contemporary, constructivist view derived from the work of Piaget, Vygotsky, Dewey and others, ${ }^{7,8}$ describes learning as being context sensitive, and this has found favour amongst medical educationalists. ${ }^{9}$ Contextualising new information to clinical situations requires a baseline resource of knowledge and experience which new undergraduates in their first years of training are unlikely to have developed. Consequently, material presented during the early phase of a programme particularly in areas that are not perceived to have immediate relevance to the practice of dentistry, might not generate the impact they deserve.
The experience of individuals related in semistructured interviews provides further qualitative evidence. In the following exchange, a recent graduate comments on anatomy teaching given during the first year:

"You study it in such great detail because obviously you do all your pre-clinical subjects in your first year and then its never revisited really in any detail so, it kind of seems that it should be something we go back to."

In contrast, when commenting on a course the following year that introduced core clinical skills the same respondent was better able to contextualise the information and noted:

"We had a term just on clinical skills (amalgam and cavities and what have you) and that seemed far more relevant."

In a second example, when asked "do you feel that part of the problem in tackling these basic science questions at an early stage was that you simply weren't aware of what was relevant and what wasn't?" The respondent gave the impression of having been somewhat overwhelmed with quantity of new information presented in the first year, the value of which was not fully appreciated:

"Yes I think so ... I think that would be fair to say. Because you are just bamboozled with all this information and you think, 'Oh Crikey, I'd best go and learn all of it!' when in actual fact if you had been a bit more focused and a bit more selective it might have made a more lasting impression."

These comments were shared by almost all those interviewed and point to their inexperience as learners leading to difficulty in contextualising information. Finally, it was interesting to note that most respondents felt that applied science was an important area with a place in postgraduate teaching at the pre-specialist level. This view was typified by the following illustrative comment from a SHO in a dental unit in large teaching hospital commenting on his/her basic medical science experience as an undergraduate:

"When you do it, you do it before you do any clinical and it doesn't really have any relevance ... they don't ever really bring it back again and say how it all relates together - its just left."

Further evidence pointing to a prevailing perception of under confidence in the application of biomedical science to clinical situations comes from analysis of other topic areas. In oral diagnostic science, oral pathology was a dominant area of perceived weakness whilst in child dental health, growth At development caused greatest concern. Both areas have their roots in biomedical science in contrast to other options which were more closely allied to clinical activity.

Other areas worried respondents for different reasons. Law and ethics, health and safety, biostatistics and epidemiology are topics where exposure varied considerably between dental schools. Considering health and safety another respondent commented:

"We didn't really have much teaching on health and safety regulations at undergraduate level. We had some IRR (ionizing radiation regulations) teaching 
but in terms of health and Safety and COSHH, we didn't have any of that teaching at all."

and in terms of law and ethics: "I just didn't feel that I was taught that very well. I think it is something that you do need to know, especially in this day and age."

Another respondent reported having no instruction or formal guidance in Health and Safety, and had been reliant instead on a video presentation. Law and ethics was covered by occasional lectures from visiting lecturers with little opportunity for discussion. Considered together, these comments suggested that teaching in these areas did not always meet individual requirements.

On beginning independent practice, subjects which appeared 'dry' at dental school suddenly become interesting and relevant. Issues relating to law and ethics, health and safety and clinical governance, which might, in the student's mind, take second place to hands-on clinical topics suddenly come to require thought and the exercise of clinical judgment. By emphasising them at the immediate postgraduate, pre-specialist phase, it may be easier to consider them in an appropriate context. MFDS level teaching has a great deal to contribute in this regard.

Given the central role of restorative dentistry in dental practice, the relatively small number of individuals expressing under-confidence in this area is notable. Restorative dentistry is central to all dental curricula and is generally well taught in settings which dental students find easy to contextualise. When highlighted, it was often by individuals actively working in the area who were happy with their command of the core skills but who wished to use preparation for the examination as an opportunity to extend them. This excerpt from an interview with a dentist working in general practice illustrates the point:

"Especially working in general practice you find that its like you've got the basic knowledge but applying it in clinical situations, in operative and prosthodontics and implants and so forth is a bit more challenging especially in more advanced cases."

There are more junior hospital posts open to dental graduates in OMFS than any other discipline, thus it was not surprising that a proportion of participants will have or were about to commence junior hospital appointments in OMFS units. Many wished to use their MFDS preparation to ensure their readiness to handle novel and complex situations they might encounter. When considering OMFS and the restorative disciplines, therefore, MFDS preparation was not being used to address areas of weakness. Instead, examination preparation was being used to gain new knowledge which would assist in tackling new challenges. This situation was quite different from that pertaining to applied basic science where individuals were using MFDS to compensate for perceived weaknesses.

In conclusion, this study has provided data pointing to which subject areas are of particular concern to candidates preparing for the MFDS examination. Basic medical and applied clinical science, law, ethics and management related issues were particularly prominent in this regard. In the case of the first two this may be due, in part, to difficulty in contextualising complex information presented in the early part of the course and in the latter, to the problems of fitting in issues requiring careful and time consuming consideration into an already crowded syllabus. In addition to revision, candidates attending courses in preparation for this examination are looking to extend their knowledge to make them better able to handle new and challenging situations that they might encounter. These factors need to be taken into account when designing revision courses to assist them.

Finally, while the focus of this paper has been on the needs of MFDS candidates it can be viewed in a much wider context as it provides a window on the needs of all dental graduates including those who decide not to specialise and, indeed, a commentary on the undergraduate dental course itself. However, as only a proportion of recent UK graduates elect to sit the MFDS examination, the extent to which the results reflect the opinion of all new graduates is unknown. Further investigation will be required if use of this approach as a commentary on the undergraduate course is to be validated.

1. General Dental Council. The First Five Years: A Framework for Undergraduate Dental Education. London, 2002. Available at www. gdc-uk.org/pdfs/first_five_years_2002.pdf - last accessed 10.02.2005

2. Berliner D C. Educational Research: The hardest science of all. Educational Researcher 2002; 31(8):18-20.

3. Dolmans D. The effectiveness of PBL; the debate continues. Some concerns about the BEME movement. Medical Education 2003: 37:1129-30.

4. Newman M. A pilot systematic review and meta-analysis on the effectiveness of problem-based learning. LTSN-01, 2003. Available at www.hebes.mdx.ac.uk/teaching/Research/PEPBL/PSR-PBL.pdf last accessed 10.02.2005

5. Light G, Cox R. Learning and Teaching in Higher Education. London, California and New Delhi: Paul Chapman Publishing, 2001.

6. Morgan A, Beaty E. The World of the Learner. In Marton F, Hounsell D. Entwistle N (eds) The Experience of Learning. Edinburgh: Scottish Academic Press, 1997

7. Duckworth E, Evans R I. Jean Piaget: The Man and His Ideas. New York: E. P. Dutton, 1973. Available at www.questia.com - last accessed 10.02.2005

8. Vygotsky LS, Luria A R. Studies On The History Of Behavior: Ape, Primitive, and Child. Golod VI, Knox J E (eds and translators) New Jersey: Lawrence Erlbaum Associates, 1993. Available at www.questia.com - last accessed 10.02.2005

9. Van der Vleuten C P M, Dolmans D H J M, Scherbier A J J A. The need for evidence in education. Medical Teacher 2000; 22: 246-50. 